\title{
Fasting plasma glucose in the first trimester is related to gestational diabetes mellitus and adverse pregnancy outcomes
}

\author{
Jia-Ning Tong ${ }^{1} \cdot{\text { Lin-Lin } \mathrm{Wu}^{1} \cdot \text { Yi-Xuan Chen }}^{1} \cdot$ Xiao-Nian Guan $^{1} \cdot$ Fu-Ying Tian ${ }^{1} \cdot$ Hua-Fan Zhang ${ }^{1} \cdot$ Kan Liu $^{1}$ • \\ Ai-Qi Yin ${ }^{1} \cdot$ Xiao-Xia Wu' ${ }^{1}$ Jian-Min Niu Prof ${ }^{1}$
}

Received: 23 April 2021 / Accepted: 16 July 2021 / Published online: 3 August 2021

(c) The Author(s) 2021

\begin{abstract}
Purpose To investigate and identify first-trimester fasting plasma glucose (FPG) is related to gestational diabetes mellitus (GDM) and other adverse pregnancy outcomes in Shenzhen population.

Methods We used data of 48,444 pregnant women that had been retrospectively collected between 2017 and 2019. Logistic regression analysis was used to evaluated the associations between first-trimester FPG and GDM and adverse pregnancy outcomes, and used to construct a nomogram model for predicting the risk of GDM. The performance of the nomogram was evaluated by using ROC and calibration curves. Decision curve analysis (DCA) was used to determine the clinical usefulness of the first-trimester FPG by quantifying the net benefits at different threshold probabilities.

Results The mean first-trimester FPG was $4.62 \pm 0.42 \mathrm{mmol} / \mathrm{L}$. A total of 6998 (14.4\%) pregnancies developed GDM.489 $(1.01 \%)$ pregnancies developed polyhydramnios, the prevalence rates of gestational hypertensive disorder (GHD), cesarean section, primary cesarean section, preterm delivery before 37 weeks (PD) and dystocia was 1130 (2.33\%), 20,426 (42.16\%), 7237 (14.94\%), $2386(4.93 \%)$, and 1865 (3.85\%), respectively. $4233(8.74 \%)$ of the newborns were LGA, and the number of macrosomia was $2272(4.69 \%)$, LBW was $1701(3.51 \%)$ and $5084(10.49 \%)$ newborns had admission to the ICU, which all showed significances between GDM and non-GDM groups (all $P<0.05$ ). The univariate analysis showed that first-trimester FPG was strongly associated with risks of outcomes including GDM, cesarean section, macrosomia, GHD, primary cesarean section, and LGA (all OR $>1$, all $P<0.05$ ), furthermore, the risks of GDM, primary cesarean section, and LGA was increasing with first-trimester FPG as early as it was at $4.19-4.63 \mathrm{mmol} / \mathrm{L}$. The multivariable analysis showed that the risks of GDM (ORs for FPG 4.19-4.63, 4.63-5.11 and 5.11-7.0 mmol/L were 1.137, 1.592, and 4.031, respectively, all $P<0.05$ ) increased as early as first-trimester FPG was at $4.19-4.63 \mathrm{mmol} / \mathrm{L}$, and first-trimester FPG which was also associated with the risks of cesarean section, macrosomia and LGA (OR for FPG 5.11-7.0 mmol/L of cesarean section: 1.128; OR for FPG 5.11-7.0 mmol/L of macrosomia: 1.561; OR for FPG 4.63-5.11 and 5.11-7.0 mmol/L of LGA: 1.149 and 1.426, respectively, all $P<0.05$ ) and with its increasing, the risks of LGA increased. Furthermore, the nomogram had a C-indices 0.771 (95\% CI: $0.763 \sim 0.779)$ and 0.770(95\% CI:0.758 0.781) in training and testing validation respectively, which showed an acceptable consistency between the observed, validation and nomogram-predicted probabilities, the DAC curve analysis indicated that the nomogram had important clinical application value for GDM risk prediction.

Conclusions FPG in the first trimester was an independent risk factor for GDM which can be used as a screening test for identifying pregnancies at risk of GDM and adverse pregnancy outcomes.
\end{abstract}

Keywords The First Trimester FPG · GDM • Metabolic Diseases in Pregnancy • Adverse Pregnancy Outcomes

Supplementary information The online version contains supplementary material available at https://doi.org/10.1007/s12020021-02831-w.

Jian-Min Niu Prof

njianmin@163.com

1 Department of Obstetrics, Shenzhen Maternity \& Child Healthcare
Hospital, The First School of Clinical Medicine, Southern Medical University, Hongli Road, Futian District, Shenzhen, Guangdong Province, China 


\section{Introduction}

Gestational diabetes mellitus (GDM) refers to an abnormality of glycometabolism that occurs for the first time in the second or third trimester of pregnancy and does not include type 1 or type 2 diabetes, which exists before pregnancy [1]. GDM is associated with adverse maternal and fetal outcomes and maternal complications in pregnancy and later in life. The prevalence of GDM is increasing; this is closely linked to the prevalence of obesity and type 2 diabetes in specific countries, and the prevalence of obesity among women of childbearing age partly explains this increase. The risks posed to mothers with GDM range from direct pregnancy complications, particularly the need for cesarean section and risk of gestational hypertension, to their lifetime risk of developing type 2 diabetes and cardiovascular diseases. Regarding their children, there is an increased shortterm risk of obesity, premature birth, shoulder dystocia, and neonatal hypoglycemia, as well as a long-term risk of obesity and abnormal plasma glucose (PG) metabolism. Therefore, GDM is associated with a particularly poor prognosis [1-3] and early detection of GDM is of great importance to help with prevention and treatment.

Epidemiological studies of hyperglycemia and adverse pregnancy outcomes (HAPO) in multiple countries have recommended that a fasting plasma glucose (FPG) value of $5.1 \mathrm{mmol} / \mathrm{L}(92 \mathrm{mg} / \mathrm{dL})$ in the first trimester can be the threshold for elevated blood glucose. It also indicated that if FPG $\leq 4.4 \mathrm{mmol} / \mathrm{L}(80 \mathrm{mg} / \mathrm{dL})$, the risks of some adverse pregnancy outcomes are low [4]. Furthermore, several researchers examined whether first-trimester FPG is also consistently associated with obstetric complications, and a retrospective study of 6129 pregnant women by Riskin-Masiah who observed first-trimester FPG found that FPG is associated with adverse outcomes and risk of GDM [5]. Therefore, it is valuable to provide more data about first-trimester FPG from a single medical database where there might be some homogeneity in the patient population. Due to metabolic changes during pregnancy, blood glucose between 6 and 10 weeks in the first trimester can drop by $\sim 2 \mathrm{mg} / \mathrm{dL}$, and many scholars have pointed out that a specific lower limit of first-trimester FPG should be defined [6].

This study hoped to provide new evidence which could identify the relationships with first-trimester FPG, GDM, and other obstetrical outcomes in the Shenzhen population.

\section{Materials and methods}

This survey was an analysis of retrospectively collected data from the clinical database of the Shenzhen Maternity and Child Healthcare System between 2017 and 2019. Patients younger than 18 years old or with incomplete information, diagnosed pregestational diabetes, multiple pregnancies, or pregnancies conceived by assisted reproductive technology were excluded. Patients included were singleton pregnancies who attended our hospital to establish a maternal-fetal manual in the first trimester, performed regular visits and gave birth in our hospital. They also received routine FPG testing in the first trimester. Finally, the selected patients included only those with an available FPG in the first trimester $(<14$ weeks $)$ performed under the standard conditions and who had complete data on all outcomes (Fig. 1). All patients were managed according to standard clinical protocols, and throughout the research periods, protocols

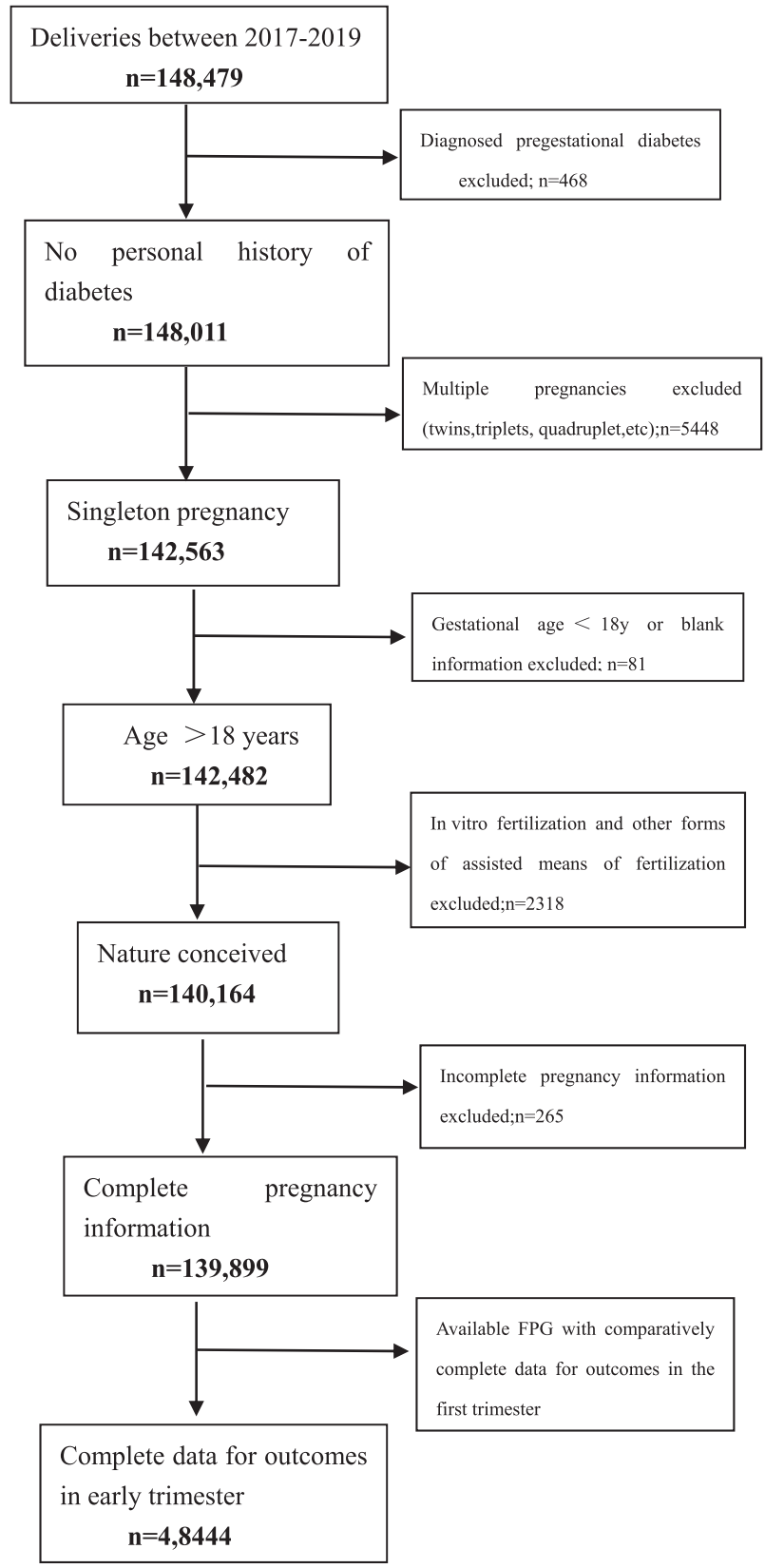

Fig. 1 Flow chart of selecting process of the survey 
were in accordance with the screening and management of GDM, followed by the recommendation of the International Diabetes and Pregnancy Research Group (IADASG) [1]. According to a previous study, the incidence of GDM in pregnant women was about $10 \sim 15 \%$, with an OR of 3.3 [7], with this assumption, 22,780 pregnant women would yield $80 \%$ power to show an incidence of GDM of $15 \%$, considering a dropout rate $20 \%$, a total sample size required for the study was 27,336 .

\section{Diagnostic criterion}

\section{Gestational diabetes mellitus (GDM)}

American Diabetes Association (ADA) has been using the one-step approach of the IADASG as the screening and diagnostic standard for GDM since 2011; here, in this study, the 2019 reviewed version was used [8]. Specifically, the IADPSG recommends that all pregnant women with no previous history of diabetes take a 75 -g oral glucose tolerance test (OGTT) at 24 to 28 gestational weeks. Any value above baseline before glucose consumption $(0 \mathrm{~h})$ or PG levels at $1 \mathrm{~h}$ and $2 \mathrm{~h}$ after glucose consumption that are abnormal were diagnosed as GDM, namely, $0 \mathrm{~h} \geq 5.1 \mathrm{mmol} /$ $\mathrm{L}(92 \mathrm{mg} / \mathrm{dl}), \quad 1 \mathrm{~h} \geq 10.0 \mathrm{mmol} / \mathrm{L} \quad(180 \mathrm{mg} / \mathrm{dl})$, and $2 \mathrm{~h} \geq$ $8.5 \mathrm{mmol} / \mathrm{L}(153 \mathrm{mg} / \mathrm{dl})$.

\section{Gestational hypertensive disorder (GHD)}

Preeclampsia was defined as systolic pressure $\geq 140 \mathrm{~mm} \mathrm{Hg}$ or diastolic pressure $\geq 90 \mathrm{~mm} \mathrm{Hg}$ on two or more occasions a minimum of $6 \mathrm{~h}$ apart, proteinuria $\geq 1+$ or more on a dipstick test, or urine protein $\geq 300 \mathrm{mg}$ for a 24 -h period. Gestational hypertension was diagnosed when elevated blood pressure met the criteria but without protein urine [9].

\section{Prepregnancy body mass index (BMI)}

To calculate BMI, prepregnancy weight $(\mathrm{kg})$ was divided by the squared height $\left(\mathrm{m}^{2}\right)$. Prepregnancy BMI was categorized according to the WHO standard [10]: women were underweight (BMI $<18.5 \mathrm{~kg} / \mathrm{m}^{2}$ ), normal $\left(18.5-25 \mathrm{~kg} / \mathrm{m}^{2}\right.$ ), overweight $\left(25-30 \mathrm{~kg} / \mathrm{m}^{2}\right)$, obese $\left.\geq 30 \mathrm{~kg} / \mathrm{m}^{2}\right)$, obese grade 1 $\left(30-35 \mathrm{~kg} / \mathrm{m}^{2}\right)$, obese grade $2\left(35-40 \mathrm{~kg} / \mathrm{m}^{2}\right)$, or obese grade $3\left(\geq 40 \mathrm{~kg} / \mathrm{m}^{2}\right)$.

\section{Gestational weight gain}

The gestational weight gain (GWG) in $\mathrm{kg}$ of the first trimester was calculated as the weight at $13^{+6}$ gestational weeks minus the prepregnancy weight. The GWG of the first trimester was categorized by the IOM (Institute of
Medicine) standard [11]: inadequate $(\mathrm{GWG}<0.5 \mathrm{~kg})$, adequate (GWG 0.5-2.0 kg), and excessive (GWGå $2.0 \mathrm{~kg}$ ).

\section{Macrosomia, large for gestational age (LGA) and low birth weight (LBW)}

Macrosomia was defined as a newborn weight in $\mathrm{g} \geq 4000$. Large for gestational age (LGA) was defined as newborn birth weight of above the 90th percentile if the birth weight was greater than the estimated 90th percentile for the same gestational age. Low birth weight (LBW) was defined as newborn birth weight $<2500 \mathrm{~g}[4,12]$.

All patients were considered when analyzing GDM and FPG in the first trimester. To analyze other obstetrical and maternal-fetal outcomes, patients with GDM were excluded to avoid bias arising from different treatments for GDM.

\section{Data collection}

We collected the descriptive statistics, clinical biochemical information, and pregnancy outcomes of the patients. The descriptive statistics referred to age, height prepregnancy BMI, etc. Pregnant women generally had their first visits at gestational weeks $9-13^{+6}$. Clinical and biochemical data were collected retrospectively from the first prenatal visit, and data about the neonatal outcomes were collected after birth and saved into standardized maternalnatal information systems for the following statistical analysis. Clinical information also covered a history of hypertension and diabetes, among other conditions. Pregnancy outcomes included complications for pregnancies and newborns.

\section{Diagnostic method}

The OGTT and FPG results were measured by using the enzyme electrode method (DXC800, Beckman). The standard laboratory procedure is to centrifuge samples within $20 \mathrm{~min}$ of collection. The results were collected retrospectively from the report system of the laboratory.

\section{Outcomes}

The obstetrical adverse outcomes included GDM, cesarean section, primary cesarean section, polyhydramnios, preterm delivery before 37weeks (PD), dystocia, and GHD, which included high blood pressure during pregnancy and preeclampsia. The neonatal outcomes included macrosomia, LGA, LBW, and ICU attendance of newborns. The main outcomes for this survey were the risk of GDM, primary cesarean section, and LGA, while the others were secondary outcomes. 


\section{Statistical analysis}

Analyses were performed using $R$ statistical software version 3.6.1. Continuous variables were presented as the means with standard deviations, while categorical data were expressed as counts and percentages. Summary statistics between both groups were compared using either unpaired Student's $t$ test or Mann-Whitney tests for continuous data, and chi-squared tests or Fisher's Exact test for categorical data. Univariate and multivariable-adjusted odds ratios (OR) with $95 \%$ confidence interval (CI) of FPG for associations between first-trimester FPG and GDM and adverse pregnancy outcomes were estimated using the logistical regression model. Nomogram and calibration curve were performed with the "rms" package, then, a nomogram diagram for predicting the risk of GDM with first-trimester FPG for GDM was established by using the stepAIC filter variables, which nomogram model was used for predicting the risk of GDM and enabling the user to easily compute output probabilities. Decision curve analysis (DCA) was performed with the "dca" package, which was conducted to determine the clinical usefulness of the first-trimester FPG nomogram by quantifying the net benefits at different threshold probabilities. A $p$ value of $<0.05$ was considered to indicate statistical significance.

\section{Results}

\section{Baseline demographic and adverse outcome}

The baseline demographic and adverse outcomes according to the presence of GDM were summarized in Table 1 . Among 148,479 pregnancies delivered between 2017 and 2019 , a total of 48,444 pregnant women were included in this study and 6,998(14.4\%) pregnancies were diagnosed as GDM. The mean maternal age was $30.85 \pm 4.04$ years, which showed a significant difference between non-GDM and GDM groups $(30.57 \pm 3.94$ vs. $32.52 \pm 4.22, P<$ 0.001 ). The pregestational BMI was $20.65 \pm 2.65 \mathrm{~kg} / \mathrm{m} 2$, which was higher in GDM groups $(20.50 \pm 2.56$ vs. $21.69 \pm$ $3.01, P<0.001)$ when compared with non-GDM groups. And first-trimester FPG was $4.62 \pm 0.42 \mathrm{mmol} / \mathrm{L}$, of which $12.18 \%$ were first-trimester $\mathrm{FPG} \leq 4.19 \mathrm{mmol} / \mathrm{L}, 73.29 \%$ were $4.19-4.62 \mathrm{mmol} / \mathrm{L}, 37.61 \%$ were $4.63-5.10 \mathrm{mmol} / \mathrm{L}$, and $9.31 \%$ were $5.11-7.0 \mathrm{mmol} / \mathrm{L}$, and the results indicated that first-trimester FPG was higher in GDM groups $(P<$ 0.001 ). For newborns, the weight of newborns was $3281.52 \pm 440.43 \mathrm{~g}$, which was a significant difference between non-GDM and GDM groups $(3284.19 \pm 434.95$ vs. $3265.74 \pm 471.30, \mathrm{P}<0.001$ ), the scores of Apgar $1 \mathrm{~min}$ and Apgar 5 min was $9.92 \pm 0.50$ and $9.99 \pm 0.63$, respectively. Four hundred and eighty-nine (1.01\%) pregnancies developed polyhydramnios, the prevalence rates of GHD, cesarean section, primary cesarean section, PD, and dystocia was $1130(2.33 \%), 20426(42.16 \%), 7237(14.94 \%)$, 2386 (4.93\%), and 1865 (3.85\%), respectively, which all showed significances between two groups (all $P<0.05$ ). Four thousand two hundred and thirty-three $(8.74 \%)$ of the newborns were LGA, and the number of macrosomia was 2272 (4.69\%), LBW was 1701 (3.51\%), and 5084 (10.49\%) newborns had admission to the ICU, all the prevalence rates were higher in the GDM group than those in the non-GDM groups (all $P<0.05$ ).

\section{Effects of first-trimester FPG on GDM and adverse outcomes}

Table 2 presented the effects of first-trimester FPG on GDM and adverse pregnancy outcomes. The univariate analysis showed first-trimester FPG was strongly associated with risks of outcomes, including GDM, cesarean section, macrosomia, GHD, primary cesarean section, and LGA (all OR $>1$, all $P<0.05$ ), furthermore, the risks of GDM, primary cesarean section, and LGA was increasing with firsttrimester FPG as early as it was at $4.19-4.63 \mathrm{mmol} / \mathrm{L}$. At the same time, first-trimester FPG was a protective factor of LBW and ICU admission of the newborn (all $\mathrm{OR}<1$, all $P$ $<0.05)$. After adjustments for multifactor, every stage of first-trimester FPG was associated with the risk of GDM (ORs for FPG 4.19-4.63, 4.63-5.11, and 5.11-7.0 mmol/L were $1.137,1.592$, and 4.031 , respectively, and $95 \%$ CIs were $1.002-1.289,1.406-1.801$, and 3.513-4.625, respectively, all $P<0.05$ ) and with increasing first-trimester FPG, the risks of GDM increased (the OR value increased). It was also associated with the risks of cesarean section, macrosomia, and LGA (OR for FPG 5.11-7.0 mmol/L of cesarean section: 1.128, 95\% CI: 1.025-1.241; OR for FPG $5.11-7.0 \mathrm{mmol} / \mathrm{L}$ of macrosomia: $1.561,95 \%$ CI: 1.26-1.933; OR for FPG 4.63-5.11 and 5.11-7.0 mmol/L of LGA: 1.149 and 1.426, 95\% CI: $1.004-1.314$ and 1.214-1.675, respectively, all $P<0.05$ ) and with its increasing, the risks of LGA increased. At the same time, first-trimester FPG was a protective factor against LBW and ICU admission of the newborn (all $\mathrm{OR}<1$, all $P<0.05$ ).

We also conducted a subgroup analysis, which revealed both the GDM and the non-GDM subgroups had similar trends. In the GDM group, first-trimester FPG was associated with the risks of macrosomia, LGA, and dystocia (all OR $>1$, all $P<0.05$ ). While in the non-GDM subgroup, FPG in the first trimester was identified as a significant predictor for the risks of cesarean section, macrosomia, and LGA (all OR $>1$, all $P<0.05$ ), and it was a protective factor against GHD, LBW, primary cesarean section, and ICU admission of the newborn (all $\mathrm{OR}<1$, all $P<0.05$ ) (Table $\mathrm{S} 1, \mathrm{~S} 2)$. 
Table 1 Baseline demographic and adverse pregnancy outcome

\begin{tabular}{|c|c|c|c|c|}
\hline Characteristics & Overall & No GDM & GDM & $P$ value \\
\hline$n$ & 48,444 & $41,446(85.6 \%)$ & $6998(14.4 \%)$ & \\
\hline \multicolumn{5}{|l|}{ Maternal Characteristics } \\
\hline Maternal age, years ${ }^{\mathrm{a}}$ & $30.85 \pm 4.04$ & $30.57 \pm 3.94$ & $32.52 \pm 4.22$ & $<0.001$ \\
\hline Height, m & $1.60 \pm 0.07$ & $1.60 \pm 0.07$ & $1.59 \pm 0.07$ & $<0.001$ \\
\hline Prepregnancy BMI, $\mathrm{kg} / \mathrm{m}^{2}$ & $20.65 \pm 2.65$ & $20.50 \pm 2.56$ & $21.69 \pm 3.01$ & $<0.001$ \\
\hline \multicolumn{4}{|l|}{ Category of prepregnancy $\mathrm{BMI}^{\mathrm{b}}(n, \%)$} & $<0.001$ \\
\hline$\leq 18.5 \mathrm{~kg} / \mathrm{m}^{2}$ & $7530(20.5)$ & $6960(21.6)$ & $570(12.4)$ & \\
\hline $18.5-24.9 \mathrm{~kg} / \mathrm{m}^{2}$ & $26,941(73.3)$ & $23,513(73.1)$ & $3428(74.5)$ & \\
\hline $25.0-29.9 \mathrm{~kg} / \mathrm{m}^{2}$ & $2099(5.7)$ & $1552(4.8)$ & $47(11.9)$ & \\
\hline $30.0-34.9 \mathrm{~kg} / \mathrm{m}^{2}$ & $163(0.4)$ & $116(0.4)$ & $47(1.0)$ & \\
\hline $35.0-39.9 \mathrm{~kg} / \mathrm{m}^{2}$ & $17(0.0)$ & $12(0.0)$ & $5(0.1)$ & \\
\hline$\geq 40.0 \mathrm{~kg} / \mathrm{m}^{2}$ & $7(0.0)$ & $5(0.0)$ & $2(0.0)$ & \\
\hline FPG in first trimester, $\mathrm{mmol} / \mathrm{L}$ & $4.62 \pm 0.42$ & $4.59 \pm 0.39$ & $4.80 \pm 0.55$ & $<0.001$ \\
\hline \multicolumn{4}{|l|}{ Category of FPG in first trimester $(\mathrm{n}, \%)$} & $<0.001$ \\
\hline$\leq 4.19 \mathrm{mmol} / \mathrm{L}$ & $5889(12.2)]$ & $5355(12.9)$ & $534(7.7)$ & \\
\hline $4.19-4.62 \mathrm{mmol} / \mathrm{L}$ & $19,770(40.9)$ & $17,621(42.6)$ & $2149(30.9)$ & \\
\hline $4.63-5.10 \mathrm{mmol} / \mathrm{L}$ & $18,184(37.6)$ & $15384(37.2)$ & $2800(40.2)$ & \\
\hline $5.11-7.0 \mathrm{mmol} / \mathrm{L}$ & $4503(9.3)$ & $3029(7.3)$ & $1474(21.2)$ & \\
\hline Delivery times & $0.37 \pm 0.53$ & $0.35 \pm 0.51$ & $0.49 \pm 0.57$ & $<0.001$ \\
\hline OGTT at $0 \mathrm{~h}, \mathrm{mmol} / \mathrm{L}^{\mathrm{d}}$ & $2.52 \pm 2.17$ & $2.42 \pm 2.14$ & $3.10 \pm 2.23$ & $<0.001$ \\
\hline OGTT at $1 \mathrm{~h}, \mathrm{mmol} / \mathrm{L}^{\mathrm{d}}$ & $4.41 \pm 3.87$ & $4.08 \pm 3.66$ & $6.35 \pm 4.51$ & $<0.001$ \\
\hline OGTT at $2 \mathrm{~h}, \mathrm{mmol} / \mathrm{L}^{\mathrm{d}}$ & $3.91 \pm 3.41$ & $3.62 \pm 3.22$ & $5.61 \pm 3.99$ & $<0.001$ \\
\hline \multicolumn{4}{|l|}{ Delivery mode $(\mathrm{n}, \%)$} & $<0.001$ \\
\hline Obstetric forceps & $282(0.6)$ & $234(0.6)$ & $48(0.7)$ & \\
\hline Eutocia & $27,294(56.3)$ & $23,799(57.4)$ & $3495(49.9)$ & \\
\hline Vacuum extraction & $424(0.9)$ & $347(0.8)$ & $77(1.1)$ & \\
\hline Breech presentation & $18(0.0)$ & $18(0.0)$ & $0(0.0)$ & \\
\hline Cesarean section & $20,426(42.2)$ & $17048(41.1)$ & $3378(48.3)$ & $<0.001$ \\
\hline Primary cesarean section & $7237(14.9)$ & $5691(13.7)$ & $1546(22.1)$ & $<0.001$ \\
\hline Gestational weight gain (GWG), Kg & $2.21 \pm 2.58$ & $2.21 \pm 2.57$ & $2.20 \pm 2.60$ & 0.768 \\
\hline \multicolumn{4}{|l|}{ Category of $\mathrm{GWG}^{\mathrm{c}}(\mathrm{n}, \%)$} & 0.264 \\
\hline$<0.5 \mathrm{~kg}$ & $6212(22.8)$ & $5280(22.7)$ & $932(23.5)$ & \\
\hline $0.5-2 \mathrm{~kg}$ & 7798 (28.6) & $6703(28.8)$ & $1095(27.7)$ & \\
\hline $0.5-2 \mathrm{~kg}$ & $13227(48.6)$ & $11296(48.5)$ & $1931(48.8)$ & \\
\hline Major birth malformation of past history $(n, \%)$ & $9062(18.7)$ & $7509(18.1)$ & $1553(22.2)$ & $<0.001$ \\
\hline Bleeding amount in $24 \mathrm{~h}, \mathrm{~mL}$ & $287.82 \pm 98.80$ & $287.68 \pm 97.77$ & $88.64 \pm 104.72$ & 0.455 \\
\hline \multicolumn{5}{|l|}{ Newborn characteristics } \\
\hline Gestational age at delivery, wk & $38.88 \pm 1.48$ & $38.93 \pm 1.47$ & $38.58 \pm 1.48$ & $<0.001$ \\
\hline Weigh of newborn, g & $281.52 \pm 440.43)$ & $284.19 \pm 434.95$ & $3265.74 \pm 471.30$ & 0.001 \\
\hline Apgar $1 \mathrm{~min}$ & $9.92 \pm 0.50$ & $9.92 \pm 0.50$ & $9.92 \pm 0.51$ & 0.228 \\
\hline Apgar 5 min & $9.99 \pm 0.63$ & $9.99 \pm 0.68$ & $9.99 \pm 0.18$ & 0.854 \\
\hline \multicolumn{3}{|l|}{ Apgar $1 \min <7(\mathrm{n}, \%)$} & 0.872 & \\
\hline No & $8215(99.54)$ & $41251(99.53)$ & $6964(99.56)$ & \\
\hline Yes & $24(0.46)$ & $193(0.47)$ & $31(0.44)$ & \\
\hline \multicolumn{5}{|l|}{ Adverse outcome } \\
\hline \multicolumn{4}{|l|}{ Polyhydramnios (\%) } & 0.530 \\
\hline No & 47,955 (98.99) & $41,033(99.00)$ & $6922(98.91)$ & \\
\hline
\end{tabular}


Table 1 (continued)

\begin{tabular}{|c|c|c|c|c|}
\hline Characteristics & Overall & No GDM & GDM & $P$ value \\
\hline Yes & $489(1.01)$ & $413(1.00)$ & $76(1.09)$ & \\
\hline GHD $(n, \%)$ & & & & 0.001 \\
\hline $\mathrm{NO}$ & $47,314(97.67)$ & $40,518(97.76)$ & $6796(97.11)$ & \\
\hline Yes & $1130(2.33)$ & $928(2.24)$ & $202(2.89)$ & \\
\hline Gestational hypertension & 301 & & & \\
\hline Preeclampsia & 829 & & & \\
\hline Cesarean section $(\%)$ & & & & $<0.001$ \\
\hline NO & $28,018(57.84)$ & $24,398(58.87)$ & $3620(51.73)$ & \\
\hline Yes & $20,426(42.16)$ & $17,048(41.13)$ & $3378(48.27)$ & \\
\hline Primary cesarean section $(\%)$ & & & & $<0.001$ \\
\hline No & $41,207(85.06)$ & $35,755(86.27)$ & $5452(77.91)$ & \\
\hline Yes & $7237(14.94)$ & $5691(13.73)$ & $1546(22.09)$ & \\
\hline $\mathrm{PD}(n, \%)$ & & & & $<0.001$ \\
\hline No & $46,058(95.07)$ & $39,520(95.35)$ & $6538(93.43)$ & \\
\hline Yes & $2386(4.93)$ & $1926(4.65)$ & $460(6.57)$ & \\
\hline Dystocia $(n, \%)$ & & & & 0.001 \\
\hline No & $46,579(96.15)$ & $39,800(96.03)$ & $6779(96.87)$ & \\
\hline Yes & $1865(3.85)$ & $1646(3.97)$ & $219(3.13)$ & \\
\hline LGA $(\%)$ & & & & $<0.001$ \\
\hline No & $44,211(91.26)$ & $37,990(91.66)$ & $6221(88.90)$ & \\
\hline Yes & $4233(8.74)$ & $3456(8.34)$ & $777(11.10)$ & \\
\hline Macrosomia (\%) & & & & 0.004 \\
\hline No & $46,172(95.31)$ & $39,550(95.43)$ & $6622(94.63)$ & \\
\hline Yes & $2272(4.69)$ & $1896(4.57)$ & $376(5.37)$ & \\
\hline $\mathrm{LBW}(\%)$ & & & & $<0.001$ \\
\hline No & $46,743(96.49)$ & $40,056(96.65)$ & $6687(95.56)$ & \\
\hline Yes & $1701(3.51)$ & $1390(3.35)$ & $311(4.44)$ & \\
\hline ICU attendance of newborns $(n, \%)$ & & & & $<0.001$ \\
\hline No & $43,360(89.51)$ & $37,283(89.96)$ & $6077(86.84)$ & \\
\hline Yes & $5084(10.49)$ & $4163(10.04)$ & $921(13.16)$ & \\
\hline
\end{tabular}

$L B W$ low birth weight, $L G A$ large for gestational age, $G H D$ gestational hypertensive disorder, $P D$ preterm delivery before 37 weeks, Dystocia: shoulder dystocia or birth injury

${ }^{\mathrm{a}}$ At delivery

${ }^{\mathrm{b}}$ Categorized by WHO standard

${ }^{\mathrm{c}}$ Categorized by Institute of Medicine standard

\section{The establishment nomogram model for predicting the risk of GDM}

Based on Table 1, maternal age, pre-pregnancy BMI, firsttrimester FPG, delivery times, delivery weeks, major birth malformation of past history, GHD, OGTT at $0 \mathrm{~h}$, OGTT at $1 \mathrm{~h}$, and OGTT at $2 \mathrm{~h}$ were all significant different from pregnancies with and without GDM, a nomogram that could predict the risk of GDM was constructed. As the dataset was divided into the training and test datasets at a ratio of 7:3. The prediction results were shown in
Fig. 2 and Fig. 3. As shown in Fig. 4, the training and testing validated $\mathrm{C}$-indices for the nomogram were 0.771 (95\%CI:0.763 0.779) and $0.770 \quad(95 \%$ CI:0.758 0.781), respectively. Additionally, the calibration curves of the nomogram model in training and testing validation were shown in Fig. 5, from which we could see that the calibration curves of both training and testing were validation close to the ideal line, indicating an acceptable consistency between the nomogram model that predicted probability and the actual observed probability. 
Table 2 OR for GDM and adverse pregnancy outcomes according to first-trimester FPG

\begin{tabular}{|c|c|c|c|c|c|}
\hline Outcomes & FPG & Crude ORs & $P$ & Adjusted ORs & $P$ \\
\hline \multirow[t]{4}{*}{ GDM } & Reference & 1 & & 1 & \\
\hline & $4.19-4.62$ & $1.223(1.107 \sim 1.351)$ & $<0.001$ & $1.137(1.002 \sim 1.289)$ & 0.046 \\
\hline & $4.63-5.10$ & $1.825(1.655 \sim 2.012)$ & $<0.001$ & $1.592(1.406 \sim 1.801)$ & $<0.001$ \\
\hline & $5.11-6.99$ & $4.880(4.378 \sim 5.439)$ & $<0.001$ & $4.031(3.513 \sim 4.625)$ & $<0.001$ \\
\hline \multicolumn{6}{|c|}{ Adverse pregnancy outcome } \\
\hline \multirow[t]{4}{*}{ Cesarean section } & Reference & 1 & & 1 & \\
\hline & $4.19-4.62$ & $1.054(0.993 \sim 1.118)$ & 0.086 & $0.984(0.916 \sim 1.057)$ & 0.656 \\
\hline & $4.63-5.10$ & 1.235 (1.163 1.312) & $<0.001$ & $1.071(0.996 \sim 1.151)$ & 0.064 \\
\hline & $5.11-6.99$ & $1.440(1.331 \sim 1.557)$ & $<0.001$ & $1.128(1.025 \sim 1.241)$ & 0.014 \\
\hline \multirow[t]{4}{*}{ GHD } & Reference & 1 & & 1 & \\
\hline & $4.19-4.62$ & $0.852(0.700 \sim 1.036)$ & 0.109 & $0.746(0.594 \sim 0.935)$ & 0.011 \\
\hline & $4.63-5.10$ & $1.021(0.841 \sim 1.240)$ & 0.831 & $0.922(0.737 \sim 1.152)$ & 0.475 \\
\hline & $5.11-6.99$ & 1.496 (1.185 1.887) & 0.001 & $1.250(0.956 \sim 1.634)$ & 0.103 \\
\hline \multirow[t]{4}{*}{ LGA } & Reference & 1 & & 1 & \\
\hline & $4.19-4.62$ & 1.251 (1.113 1.406) & $<0.001$ & $1.090(0.952 \sim 1.247)$ & 0.213 \\
\hline & $4.63-5.10$ & $1.549(1.380 \sim 1.740)$ & $<0.001$ & $1.149(1.004 \sim 1.314)$ & 0.044 \\
\hline & $5.11-6.99$ & $2.124(1.853 \sim 2.436)$ & $<0.001$ & 1.426 (1.214 1.675) & $<0.001$ \\
\hline \multirow[t]{4}{*}{ Polyhydramnios } & Reference & 1 & & 1 & \\
\hline & $4.19-4.62$ & $0.841(0.639 \sim 1.106)$ & 0.216 & $0.830(0.614 \sim 1.123)$ & 0.228 \\
\hline & $4.63-5.10$ & $0.780(0.589 \sim 1.032)$ & 0.082 & $0.757(0.555 \sim 1.033)$ & 0.080 \\
\hline & $5.11-6.99$ & $0.971(0.677 \sim 1.393)$ & 0.874 & $0.971(0.653 \sim 1.442)$ & 0.883 \\
\hline \multirow[t]{4}{*}{ Dystocia } & Reference & 1 & & 1 & \\
\hline & $4.19-4.62$ & $0.980(0.843 \sim 1.140)$ & 0.793 & $1.081(0.909 \sim 1.286)$ & 0.379 \\
\hline & $4.63-5.10$ & $1.006(0.864 \sim 1.171)$ & 0.941 & $1.164(0.977 \sim 1.388)$ & 0.090 \\
\hline & $5.11-6.99$ & $0.946(0.772 \sim 1.160)$ & 0.593 & $1.187(0.940 \sim 1.500)$ & 0.150 \\
\hline \multirow[t]{4}{*}{ Primary Cesarean Section } & Reference & 1 & & 1 & \\
\hline & $4.19-4.62$ & 1.183 (1.081 1.294) & $<0.001$ & $0.893(0.780 \sim 1.022)$ & 0.101 \\
\hline & $4.63-5.10$ & $1.532(1.401 \sim 1.674)$ & $<0.001$ & $0.998(0.873 \sim 1.142)$ & 0.982 \\
\hline & $5.11-6.99$ & $1.819(1.631 \sim 2.028)$ & $<0.001$ & $0.977(0.828 \sim 1.154)$ & 0.786 \\
\hline \multirow[t]{4}{*}{ ICU attendance } & Reference & 1 & & 1 & \\
\hline & $4.19-4.62$ & $0.879(0.801 \sim 0.964)$ & 0.006 & $0.887(0.791 \sim 0.995)$ & 0.041 \\
\hline & $4.63-5.10$ & $0.890(0.810 \sim 0.977)$ & 0.014 & $0.887(0.789 \sim 0.997)$ & 0.044 \\
\hline & $5.11-6.99$ & $0.943(0.834 \sim 1.067)$ & 0.353 & $0.918(0.787 \sim 1.070)$ & 0.274 \\
\hline \multirow[t]{4}{*}{ Macrosomia } & Reference & 1 & & 1 & \\
\hline & $4.19-4.62$ & $1.259(1.076 \sim 1.473)$ & 0.004 & $1.117(0.932 \sim 1.338)$ & 0.232 \\
\hline & $4.63-5.10$ & $1.518(1.299 \sim 1.773)$ & $<0.001$ & $1.172(0.978 \sim 1.405)$ & 0.085 \\
\hline & $5.11-6.99$ & 2.067 (1.721 2.481) & $<0.001$ & $1.561(1.260 \sim 1.933)$ & $<0.001$ \\
\hline \multirow[t]{4}{*}{ LBW } & Reference & 1 & & 1 & \\
\hline & $4.19-4.62$ & $0.768(0.665 \sim 0.888)$ & $<0.001$ & $0.771(0.613 \sim 0.970)$ & 0.026 \\
\hline & $4.63-5.10$ & $0.711(0.613 \sim 0.824)$ & $<0.001$ & $0.718(0.567 \sim 0.909)$ & 0.006 \\
\hline & $5.11-6.99$ & $0.760(0.620 \sim 0.930)$ & 0.008 & $0.734(0.533 \sim 1.012)$ & 0.059 \\
\hline \multirow[t]{4}{*}{ PD } & Reference & 1 & & 1 & \\
\hline & $4.19-4.62$ & $1.021(0.890 \sim 1.171)$ & 0.768 & $1.559(0.589 \sim 4.125)$ & 0.371 \\
\hline & $4.63-5.10$ & $1.069(0.931 \sim 1.227)$ & 0.344 & $1.300(0.486 \sim 3.482)$ & 0.601 \\
\hline & $5.11-6.99$ & $1.170(0.981 \sim 1.396)$ & 0.081 & $2.232(0.673 \sim 7.406)$ & 0.190 \\
\hline
\end{tabular}

Reference: First category of early FPG $\leq 4.19 \mathrm{mmol} / \mathrm{L}$ as the reference

Adjusted ORs: adjusted by maternal age, prepregancy BMI, height, delivery times, and delivery weeks

Bold: ORå $1, P<0.05$; Italics: $\mathrm{OR}<1, P<0.05$

aFPG: FPG in the first-trimester

\section{Decision curve analysis used to evaluate prediction models}

The DCA was used to evaluate prediction models from the perspective of first-trimester FPG consequences, which revealed that compared with the conventional staging systems, the nomogram-yielded superior net clinical benefit whose threshold probabilities ranged from 0.1 to 0.6 in both the training validation and testing validation (Fig. 6). The DAC curve analysis in this clinical validity suggested that if 
Fig. 2 Nomogram model for predicting the risk of GDM in training validation

Nomogram for predicting the risk of GDM in Training validation

Points
Major.malformation
EarlyBMI
EarlyFPG
Delivery.times
Delivery.weeks
Maternal.age
OGTTOh
OGTT1h
OGTT2h
Total Points
Linear Predictor
Prediction Probility
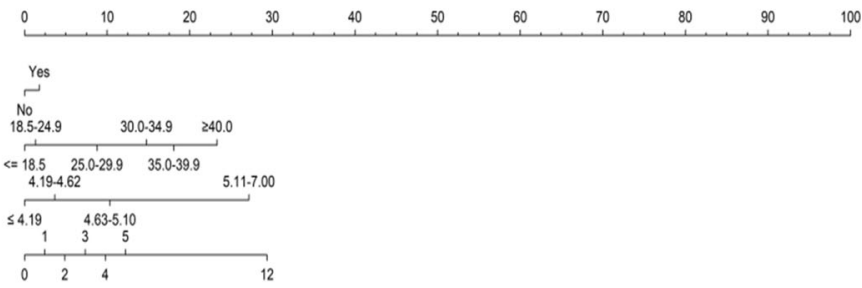

\begin{tabular}{llllllllll}
\hline 42 & 10 & 38 & 1 & 1 & 1 & 1 & 1 & 1 & 1 \\
40 & 36 & 32 & 30 & 28 & 26 & 24
\end{tabular}

$\begin{array}{llllllll}15 & 20 & 25 & 30 & 35 & 40 & 45 & 50\end{array}$

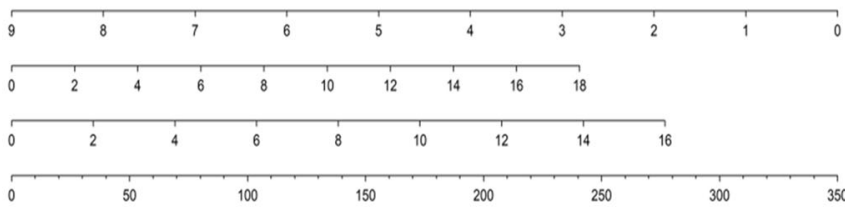

$+50$

$\begin{array}{lllllllllllllllllllll}-7 & -6 & -5 & -4 & -3 & -2 & -1 & 0 & 1 & 2 & 3 & 4 & 5 & 6 & 7\end{array}$

$\begin{array}{llllllll}0.001 & 0.01 & 0.1 & 0.3 & 0.5 & 0.7 & 0.9 & 0.99\end{array}$

Nomogram for predicting the risk of GDM in Testing validation

Points

Major.malformation

EarlyBMI

EarlyFPG

Delivery times

Delivery.weeks

Maternal.age

OGTTOH

OGTT1h

OGTT2h

Total Points

Linear Predictor

Prediction Probility
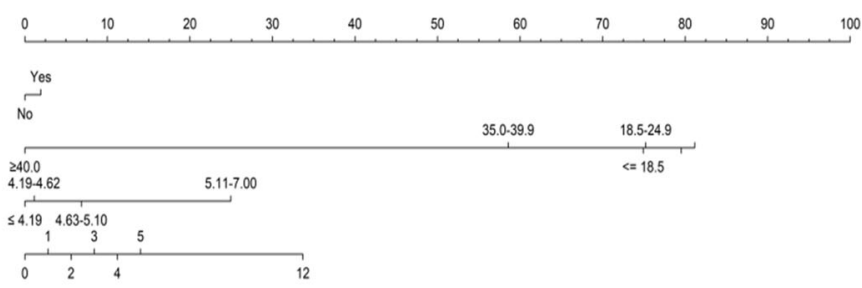

$\begin{array}{llllllllll}42 & 40 & 38 & 36 & 34 & 32 & 30 & 28 & 26 & 24\end{array}$
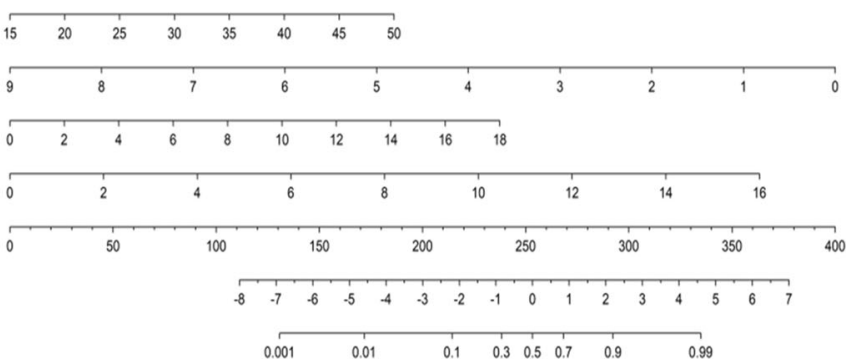

macrosomia, primary cesarean section, and LGA can increase as early as when first-trimester FPG was at 4.19-4.63 mmol/L, and with increasing first-trimester FPG, the risks of adverse outcomes increased (the OR value increased). Furthermore, we creatively used statistical models to demonstrate that the first-trimester FPG can be used to predict GDM. The nomogram showed an acceptable consistency between the observed, validation, and nomogram-predicted probabilities, the DAC curve analysis indicated that the nomogram had important clinical application value for GDM risk prediction. The above results demonstrate that the first-trimester FPG could be used to
This survey shows that in Shenzhen population, firsttrimester FPG was not only strongly associated with GDM but also with other adverse pregnancy outcomes. In the univariate and multivariable analysis, the risks of GDM, 

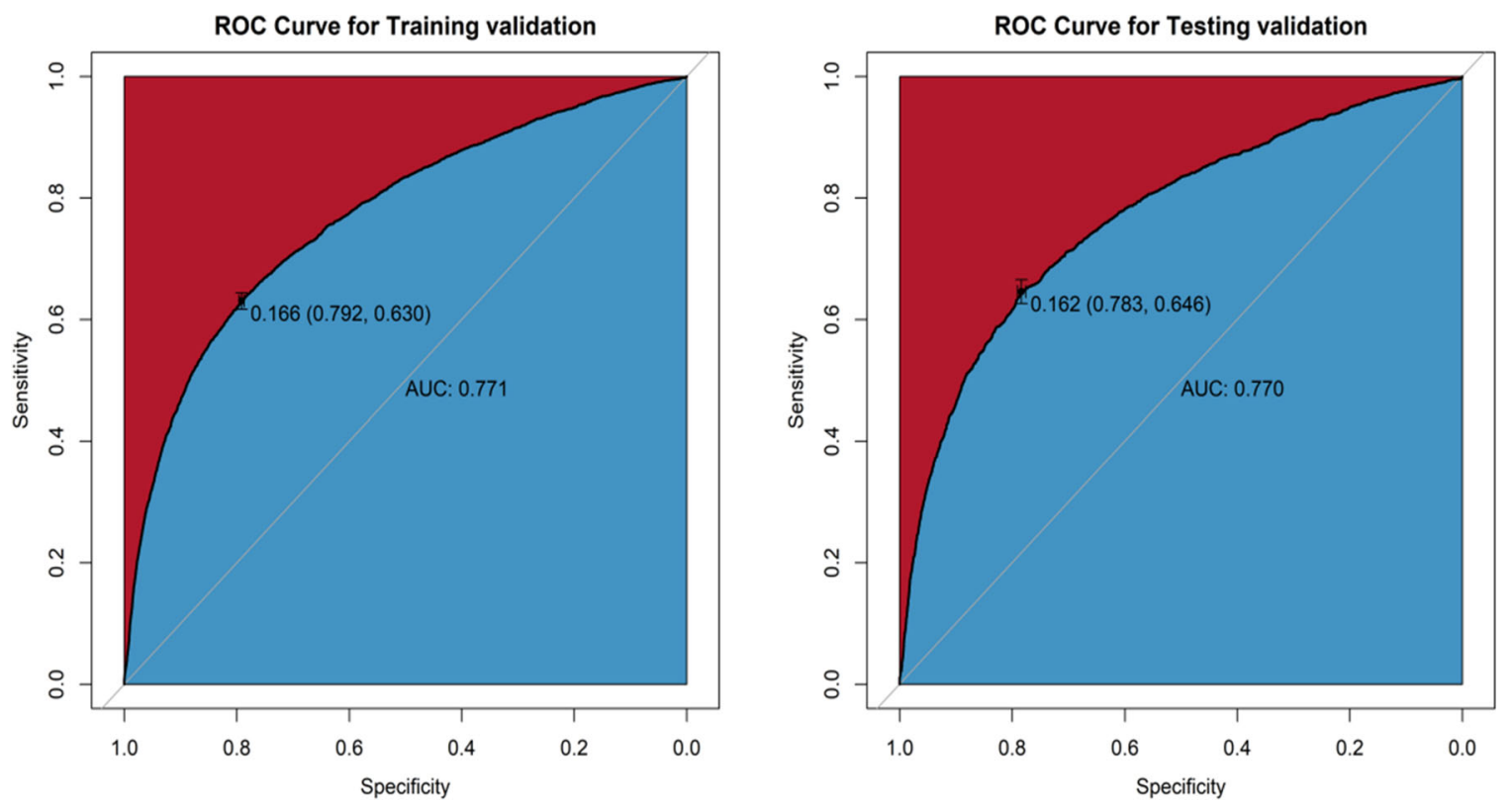

Fig. 4 The ROC curves for predictions of the risk of GDM in the training and testing validation

Calibration Curve in Training validation

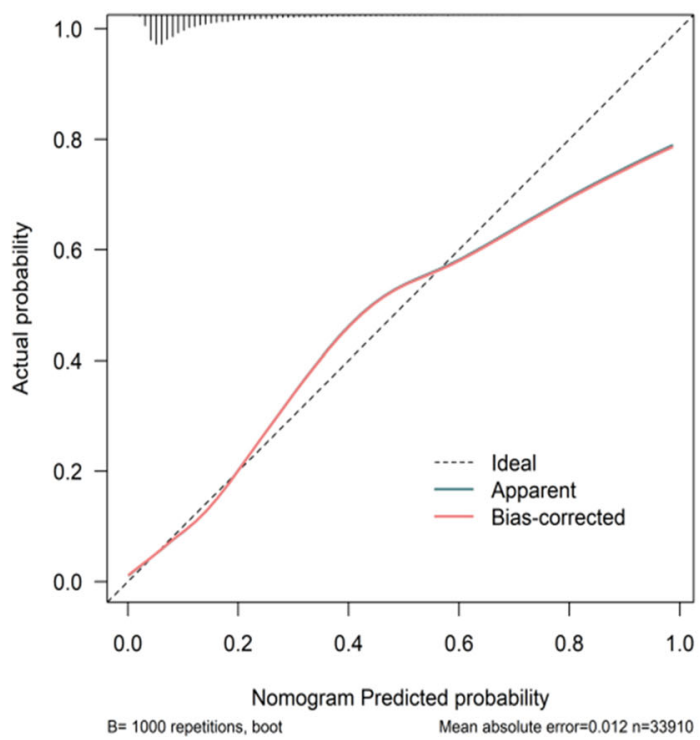

Calibration Curve in Testing validation

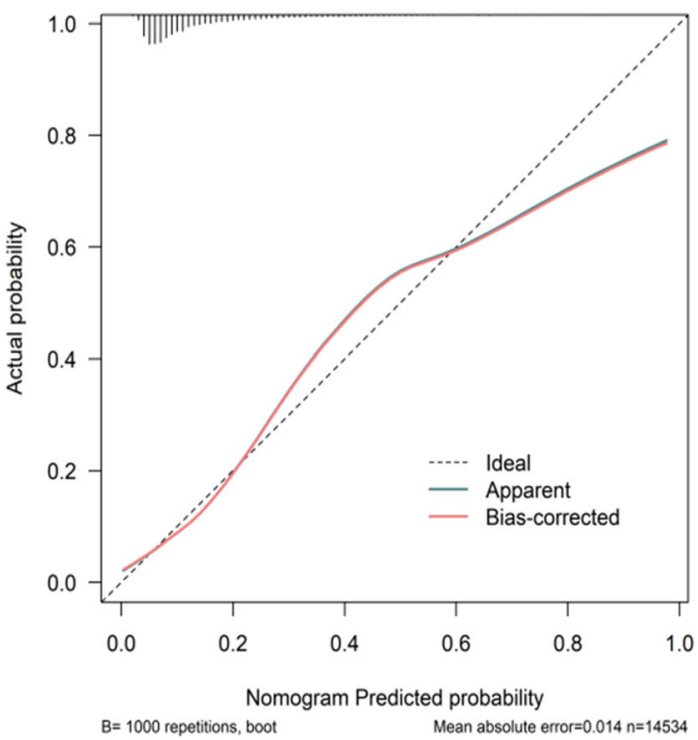

Fig. 5 The calibration curves for predictions of the risk of GDM in the training and testing validation

identify adverse pregnancy risks and intervene as early as possible due to the specific metabolic changes during pregnancy.

HAPO was a prospective observational study of 25,505 pregnant women, which showed that maternal FPG is associated with increased birth weight and primary cesarean section [4, 12]. The maternal metabolic state in the first trimester may affect the outcomes of the mothers and newborns. Riskin-Mashiah et al. reported that the mild increased levels of FPG in the first trimester can lead to adverse outcomes, and they found a strong correlation between the first-trimester FPG and GDM development [5]. We also demonstrated a consistent correlation between FPG and adverse obstetric outcomes in non-GDM patients, similar to the HAPO study [4]. Our study showed that the FPG results of HAPO also applied equally to our database in Shenzhen, China. In addition, higher first-trimester FPG, though below the diagnostic FPG criterion, was associated 

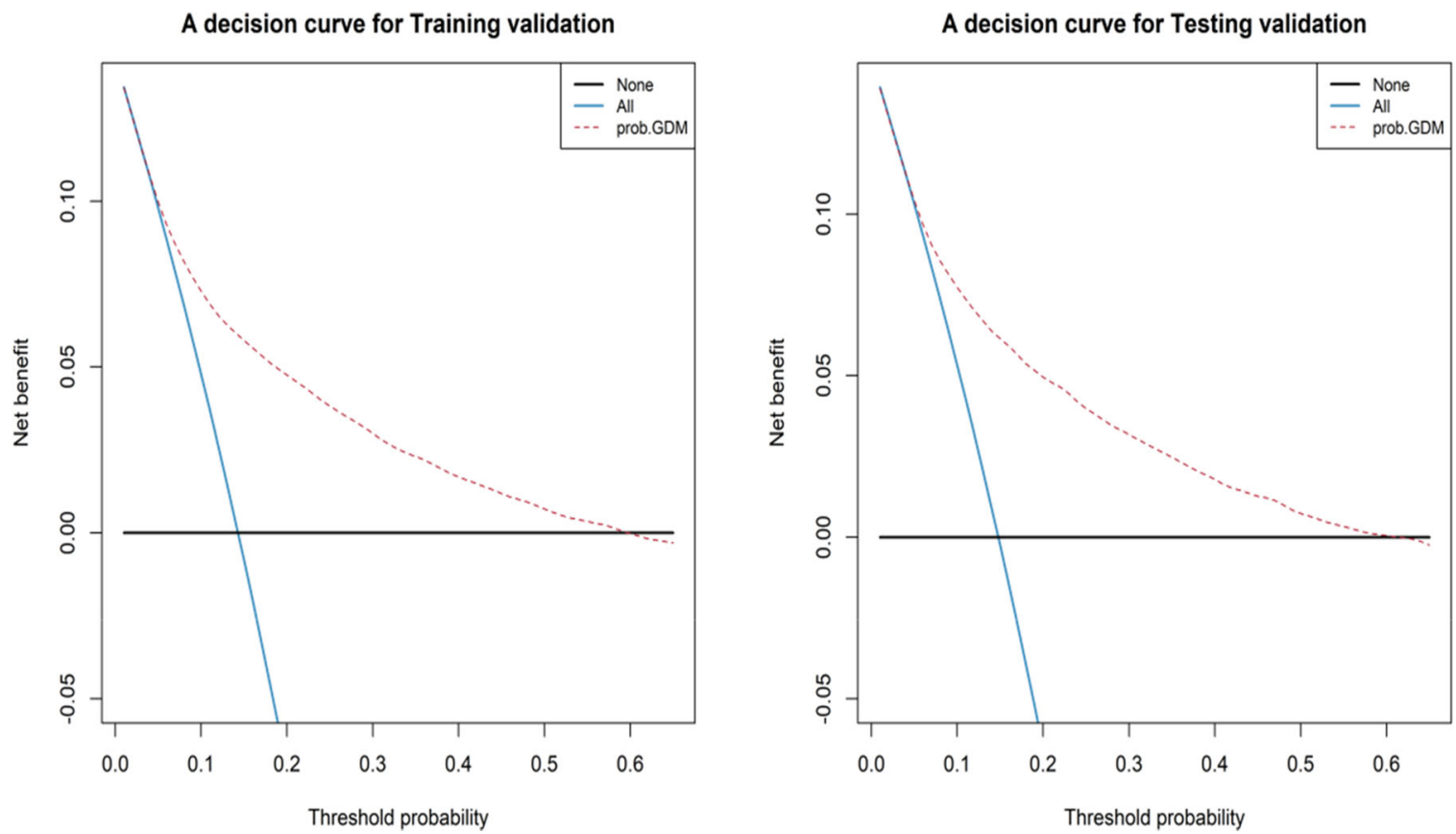

Fig. 6 Decision curve analysis for predictions of the risk of GDM in the training and testing validation

with adverse pregnancy outcomes. In our study, as early as when first-trimester FPG was in the range of $4.19-4.63 \mathrm{mmol} / \mathrm{L}$, the risks of GDM appeared, which may be a clue of the risks of GDM.

According to the ADA, GDM is a kind of diabetes diagnosed in the middle or late stages of pregnancy, and its symptoms are not obvious before pregnancy [8]. However, the diagnosis of GDM remains a controversial issue with multiple diagnostic criteria existing; its importance lies in its association with maternal and child health in pregnancy and later life [1]. Furthermore, it is agreed that GDM, regardless of symptoms, is associated with a significant risk of adverse perinatal outcomes. Several studies have shown that addressing GDM as early as possible can improve outcomes $[1,4]$, but there is much debate about its diagnosis and treatment. The main controversy involves the importance of FPG or the OGTT in the first trimester, and addressing the biases of first-trimester FPG to improve adverse outcomes for the future health of mothers and newborns stills remains discussion $[1,4]$. In the study by Sacks et al., it was indicated that FPG screening for detecting early GDM was less specific, but the AUC was 0.7, which suggested that FPG still had diagnostic accuracy in predicting GDM (AUC > 0.5 ) [6]. Zhu et al. conducted a study of 17,186 pregnancies in China by using the IDPSG standard which showed a strong correlation between the first-trimester FPG and GDM diagnosed at 24-28 weeks of pregnancy [7]. In our research, it was also found that the diagnostic model (AUC was around 0.770) of first-trimester FPG in predicting GDM had a similar trend when using the IDPSG standard. The HAPO study indicated that there is a linear relationship between maternal FPG and macrosomia, which was similar with our study.

On the other hand, there is growing evidence showing that the first-trimester FPG is a sign of maternal and newborn health. HAPO indicated that first-trimester FPG can be used to stratify the risks and set intervention thresholds. It also showed that in the one-step OGTT, the risks of birth weight, 90th percentile of $\mathrm{C}$ peptides, neonatal hypoglycemia, and primary cesarean section increased linearly as the FPG of mothers increased [5, 13]. Some of our findings were accordance with the HAPO results. However, the effectiveness of FPG in predicting GDM is not generally accepted because the diagnostic criteria vary and the choice of gestational week or race is different [4, 12]. Previous studies have shown that FPG can be used to predict the risk of diabetes in later trimesters [2, 3, 13]. Riskin-Mashiah et al. studied a large number of pregnant women from Israel $(n=6129)$ and obtained similar results to ours, namely, that first-trimester FPG has an independent relationship with the risks of GDM and LGA [5].In addition, studies of lifestyle interventions to prevent GDM have shown that it works best in the early stages of pregnancy $[14,15]$.

The search for optimal first-trimester FPG is critical in order to avoid a comprehensive OGTT test, which needs our further investigation. Based on the IADPSG standard, Agarwal et al. pointed out that FPG had shown to be a better diagnostic tool for GDM (AUC: 0.907), and $80 \mathrm{mg} / \mathrm{dL}$ $(4.44 \mathrm{mmol} / \mathrm{L})$ is recommended as the cutoff value for FPG because of its good sensitivity (95.4\%) [16]. The AUC 
calculated by Zhu et al. was 0.836 and the sensitivity was $87.8 \%$. which also indicated that the first-trimester FPG of $80 \mathrm{mg} / \mathrm{dL}$ (4.44 mmol/L) is a good predictor for GDM [17]. The results by Reyes et al. showed that the cutoff value of FPG of $90 \mathrm{mg} / \mathrm{dL}(5.0 \mathrm{mmol} / \mathrm{L})$ was helpful to avoid unnecessary OGTT in $0.7 \%$ of pregnant women [18]. Different studies all highlighted the usefulness and importance of using FPG alone for the diagnosis of GDM.

\section{Limitations}

Our research has some limitations. The nutritional status of pregnancies may affect fetal growth and other perinatal outcomes, but we lack the related data. The value of PG and bilirubin of newborns is also very important for the survey, but the data are unavailable now. A number of confounded factors, such as the delivery history of microsomia, may influence the clinical decision, such as the choice of delivery method. The lack of an identified cutoff value for firsttrimester FPG to date needs further exploration and indicates that the survey needs additional, more thorough research. Furthermore, first-trimester FPG is usually tested only once in the first prenatal examination, and its accuracy may be low. Our study evaluated the first-trimester FPG in a large number of pregnant women for the diagnosis of GDM, but the results may not be applicable to the general population of different races. Therefore, in future studies, it is strongly recommended to investigate the prevalence and different diagnostic methods of GDM after adjusting for all the possible associated factors in a large, multicenter population to increase the external validity of the results. Although there is no uniform optimal cutoff value for the first-trimester FPG to predict GDM, it was indicated that the diagnostic accuracy of FPG for predicting GDM was similar when using the IADPSG standard. Different studies have reported different levels of FPG for diagnosing GDM, which may be due to the study population, ethnicity, and diagnostic criteria $[16,19,20]$. The optimal cutoff value of FPG, especially in the first trimester, needs deeper and further study.

\section{Conclusion}

Our study did find a strong correlation between firsttrimester FPG and GDM, LGA, and other adverse obstetric outcomes, and identify its clinical significance in Shenzhen population, which suggested a need to reconsider the current criteria for diagnosing GDM and deal with higher firsttrimester FPG. Furthermore, our study complemented the literature and suggested that first-trimester FPG may be a valuable tool for predicting GDM in southern China. Based on our study, we recommend first-trimester FPG can be used to predict GDM by the IADPSG standard, as well as a marker of obstetric risk, but the diagnosis of GDM may need comprehensive consideration. The current diagnostic standards for GDM in China should be re-examined. Further research to find the optimal cutoff value for firsttrimester FPG of diagnosing GDM will be challenging.

\section{Code availability}

The codes used during and/or analyzed during the current study are available from the corresponding authors (JM Niu) on reasonable request.

Acknowledgements The authors thank Prof. Xin Zhou at Tianjin University General Hospital about the structure suggestion of the manuscript.

Author contributions J.M.N. was responsible for the study conception and design. J.N.T. did the statistical analysis and wrote the script. L.L.W., Y.X.C., X.N.G., and X.X.W. collected the data. F.Y.T. and H.F.Z. gave help to the data interpretation. K.L. and A.Q.Y. helped to revise part of the script. All authors contributed to the study design, including data collection, data interpretation, and manuscript revision, and gave final approval of the version to be published.

Funding The survey was supported by the National Natural Science Foundation of China(81830041,81771611), Shen Zhen Science and Technology Innovation Committee Special Funding for Future Industry (JCYJ20170412140326739).

\section{Compliance with ethical standards}

Conflict of interest The authors declare no competing interests.

Ethics approval This research was approved by the Review Board for Human Investigation and the Ethics Committee of Shenzhen Maternal and Children Hospital. Informed consent was obtained from every patient, and the investigations were performed in accordance with the principles of the Declaration of Helsinki.

Informed consent Written informed consent was obtained from individual or guardian participants.

Publisher's note Springer Nature remains neutral with regard to jurisdictional claims in published maps and institutional affiliations.

Open Access This article is licensed under a Creative Commons Attribution 4.0 International License, which permits use, sharing, adaptation, distribution and reproduction in any medium or format, as long as you give appropriate credit to the original author(s) and the source, provide a link to the Creative Commons license, and indicate if changes were made. The images or other third party material in this article are included in the article's Creative Commons license, unless indicated otherwise in a credit line to the material. If material is not included in the article's Creative Commons license and your intended use is not permitted by statutory regulation or exceeds the permitted use, you will need to obtain permission directly from the copyright holder. To view a copy of this license, visit http://creativecommons. org/licenses/by/4.0/. 


\section{References}

1. International Association of Diabetes and Pregnancy Study Groups Consensus Panel, International association of diabetes and pregnancy study groups recommendations on the diagnosis and classification of hyperglycemia in pregnancy. Diabetes Care. 33(3), 676-682 (2010)

2. G. Sesmilo et al, First-trimester fasting glycemia as a predictor of gestational diabetes (GDM) and adverse pregnancy outcomes. Acta Diabetol. 57(6), 697-703 (2020)

3. M.E. Bianco et al, Hyperglycemia and adverse pregnancy outcome follow-up study: newborn anthropometrics and childhood glucose metabolism. Diabetologia. 64(3), 561-570 (2021)

4. B.E. Metzger et al, Hyperglycemia and adverse pregnancy outcomes. N. Engl. J. Med. 358(19), 1991-2002 (2008)

5. S. Riskin-Mashiah, A. Damti, G. Younes, R. Auslender, First trimester fasting hyperglycemia as a predictor for the development of gestational diabetes mellitus. Eur. J. Obstet. Gynecol. Reprod. Biol. 152(2), 163-167 (2010)

6. D.B. Sacks, D.E. Bruns, D.E. Goldstein, N.K. Maclaren, J.M. McDonald, M. Parrott, Guidelines and recommendations for laboratory analysis in the diagnosis and management of diabetes mellitus. Clin. Chem. 48(3), 436-472 (2002)

7. W.W. Zhu et al, Evaluation of the value of fasting plasma glucose in the first prenatal visit to diagnose gestational diabetes mellitus in china. Diabetes Care 36(3), 586-590 (2013)

8. ADA, Standards of medical care in diabetes-2019 abridged for primary care providers. Clin. Diabetes. 37(1), 11 (2019)

9. M.A. Brown et al, Hypertensive disorders of pregnancy: ISSHP classification, diagnosis, and management recommendations for international practice. Pregnancy Hypertens. 72(1), 24-43 (2018)

10. WHO. Obesity: preventing and managing the global epidemic. World Health Organ. Tech. Rep. Ser. 894, i-xii, 1-253 (2000)

11. K.M. Rasmussen et al. (eds), Weight Gain During Pregnancy: Reexamining the Guidelines (National Academies Press, Washington DC, 2009)
12. W.L. Lowe et al, Hyperglycemia and adverse pregnancy outcome follow-up study (HAPO FUS): maternal gestational diabetes mellitus and childhood glucose metabolism. Diabetes Care 42(3), 372-380 (2019)

13. S. Riskin-Mashiah, et al., First-trimester fasting hyperglycemia and adverse pregnancy outcomes. Diabetes Care 32(9), 1639-1643 (2009)

14. E. Cosson et al., Prognosis associated with initial care of increased fasting glucose in early pregnancy: a retrospective study. Diabetes Metab. 47(3), 101197 (2021)

15. S.B. Koivusalo et al., Gestational diabetes mellitus can be prevented by lifestyle intervention: the Finnish Gestational Diabetes Prevention Study (RADIEL): a randomized controlled trial. Diabetes Care. 39(1), 24-30 (2016)

16. M.M. Agarwal et al., Gestational diabetes mellitus: simplifying the international association of diabetes and pregnancy diagnostic algorithm using fasting plasma glucose. Diabetes Care. 33(9), 2018-2020 (2010)

17. W.W. Zhu et al., Fasting plasma glucose at $24-28$ weeks to screen for gestational diabetes mellitus: new evidence from China. Diabetes Care. 36(7), 2038-2040 (2013)

18. E. Reyes-Muñoz, N.L. Sandoval-Osuna, C. Reyes-Mayoral et al., Sensitivity of fasting glucose for gestational diabetes mellitus screening in Mexican adolescents based on International Association of Diabetes and Pregnancy Study Groups criteria: a diagnostic accuracy study based on retrospective data analysis. BMJ Open 8(4), e021617 (2018)

19. M. Li et al.,Diagnostic accuracy of fasting plasma glucose as a screening test for gestational diabetes mellitus: a systematic review and meta-analysis. Eur. Rev. Med. Pharmacol. Sci. 24(21), $11172-11186$ (2020)

20. C. Nachtergaele, et al., COVID-19 pandemic: can fasting plasma glucose and HbAlc replace the oral glucose tolerance test to screen for hyperglycaemia in pregnancy? Diabetes. Res. Clin. Pract. 172, 108640 (2021) 